\title{
Industry 5.0: The Convergence of Al and HI (Human Intelligence)
}

Gauri Malhar Bhandurge ( $\nabla$ gauribhandurge@gmail.com )

COEP: Government College of Engineering

Mrunmayi Shirish Bhide

COEP: Government College of Engineering

\section{Survey paper}

Keywords: Industry 5.0, Artificial Intelligence, Industrial Upcycling, Industrial Revolution, Automation, Digitization, Cobots - Collaborative robots, Personalization, Individualization, User-friendly, Smart Homes, Smart Factory, Smart City.

Posted Date: July 14th, 2021

DOI: https://doi.org/10.21203/rs.3.rs-693806/v1

License: (c) (i) This work is licensed under a Creative Commons Attribution 4.0 International License. Read Full License 


\title{
Industry 5.0: The convergence of AI and HI (Human Intelligence)
}

\author{
Author's Name:
}

* Author 1

Author 2

*Gauri Malhar Bhandurge

Mrunmayi Shirish Bhide

* gauribhandurge@gmail.com

msbhide7@gmail.com

\author{
DEPARTMENT OF MANUFACTURING ENGINEERING AND INDUSTRIAL MANAGEMENT \\ COLLEGE OF ENGINEERING, PUNE (COEP) - MAHARASHTRA, INDIA
}

KEYWORDS: Industry 5.0, Artificial Intelligence, Industrial Upcycling, Industrial Revolution, Automation, Digitization, Cobots - Collaborative robots, Personalization, Individualization, User-friendly, Smart Homes, Smart Factory, Smart City.

\begin{abstract}
Today, as we see a big chunk of the industries in various fields adapting Industry 4.0, a lot of us have started asking the question "what's next?", the next industrial revolution, Industry 5.0. Past revolutions have pointed out that new revolutions take place only to correct the anomalies of the existing industrial situations. Likewise, to generate further clarity and to understand the previous findings around smart factory, we conducted a systematic literature review. This research paper talks about the various walks of life that industry 5.0 has the capability to affect and make better. We have also attempted to list down a few factors that will be vital for bringing about the $5^{\text {th }}$ industrial revolution. With the goal of a smart society, which will be an extension of the existing smart factory, we call this process of transformation, growth and development as 'THE INDUSTRIAL UPCYCLING'. We are proposing a new terminology called IoE - Internet of Everything as an extension of IoT.
\end{abstract}

\section{Introduction}

Bringing human intelligence and artificial intelligence together collaboratively, and not against each other, is the crux of the fifth industrial revolution. The revolution aims at being beneficial to both involved parties. This article attempts to make the idea of Industry 5.0 easy, understandable and as realistic as possible from the common man's point of view. Industry 5.0 will differ from Industry 4.0 majorly because now people will not worry losing their job to a robot, or getting their privacy invaded by a robot, solving the Human-AI conundrum.

\section{Critique of industry 4.0}


We believe that the fact that discussions regarding the next industrial revolution are already under way, even when Industry 4.0 has not been implemented globally and fully, suggests that it had its shortcomings.

A lot of philosophers believe that industry 4.0 lacks creativity. They feel that there is nothing new about the IT-

Human collaboration. In a way, they fear that this will make the IT firms richer and bigger and the common man will feel threatened.

Industry 5.0 has the primary goal of working against this fear and misconception and make people believe that they can succeed not in spite of robot presence but because of it.

\section{Current state of Industry 4.0 and marching towards Industry 5.0}

It is crucial to understand that the following prerequisites need to be fulfilled in order to bring about the above said transformation:

- Production shouldn't come to a halt even during the transition period from 4.0 to 5.0.

- The capital requirements should be fulfilled one at a time, and financial overburden should be avoided.

- Cyber security should be ensured throughout.

Along with this, the company must also understand that this change will take ample amount of time and investment and that the current scenario would not remain the same. We expect that, Industry 5.0 will create new jobs, and leadership responsibilities, namely: a General Robotics Leader (GRL), Machine learning researcher, Data scientist and a dedicated AI Product manager. [1] Recruiting the right people by keeping up the temperament, the skills, the efforts and the attitude should be considered as the primitive requirement for this transition to take place.

It is obvious that these criteria are hard to fulfill, therefore only some 'forts' of the Industry 4.0 concept have been conquered and when the condition of Industry 4.0 is such, the concept of Industry 5.0 seems far out of reach. Hence, with a view to change this situation and perspective and to bring about a feasible, good-for-all option, Industry 5.0 will prove to be a game-changer by bringing out the best from each revolution, combining it with technology to achieve the desired vision of the future.[2]

\section{An evolution of revolutions}

1. $\mathbf{1}^{\text {st }}$ industrial revolution (Mechanization): This began in 1780 when people started using steam and water to generate mechanical power.

2. $\mathbf{2}^{\text {nd }}$ industrial revolution (Electrification): This happened in 1870 and it brought the advent of electricity.

3. $\mathbf{3}^{\text {rd }}$ industrial revolution (Automation): Happened in 1970 with the introduction of machine replacement for man.

4. $4^{\text {th }}$ industrial revolution (Digitization): Began in 2011 and still continues to enthral us with everything from computers to toasters being digitally linked to each other. [6]

\section{Collaborative Robots Ushering in Industry 5.0}

The focus of Industry 4.0 was ensuring constancy of flow and quality. While Industry 5.0 focuses on highly skilled human brains and an AI robot working together to create personalized, customized and singularized products from refrigerators to cars, and also on the above-mentioned factors. Having said this, mass production and efficiency should also be considered. The concept of Digital Twins -a simulation of a process, product or service, give 
manufacturing units and other users a chance to experience a real-life situation without actually having to pay for any of the resources required. Once the simulation is successful and without any risks, it can be implemented. This avoids wastage of funds and saves production downtime.

In Industry 4.0, robots and humans worked individually and independently. They worked on the same station but there were clear demarcations between what the robot did and what the human did. As previously mentioned, with Industry 5.0, this demarcation gets blurred and an effort is being made so that the cognitive skills of the human brain can be complemented by the robotic assistance.

Collaborative robots or "cobots" are a vital element for the advent of industry 5.0 and the smart factory. These bots are designed to work alongside their human counterparts, and more importantly to assist them. They are userfriendly and their key function is to provide physical assistance to operators by carrying out the unpleasant and risky activities. With the introduction cobots there shouldn't be a fear of losing the production line to automation which has been a major concern of Industry 4.0. All of this adds agility to a firm.

Another real-time use of these technology and data driven innovations that make Industry 5.0 what it is, is in the form of Shopfloor Trackers. They track the production process at real-time which leads to reduction of waste material, and mismanagement.

\section{Industry 5.0: Encompassing Medicine}

Widening our scope of Industry 5.0 further and not just limiting it to Industries and Manufacturing, the field of medicine is also witnessing some revolutionary changes as a result of this new emerging era and this can be treated as a by-product of the blessings of Industry 5.0. All the services and technologies we see today is because of the enormous digitization that was proposed by Industry 4.0. But consequently, this concept was found to be lacking a human touch or a customer centric approach. In this context, the partnership of HI along with AI has enabled us to diagnose a patients' illness, synthesize a proper treatment for it, monitor the responses of the body to the given treatment and then constantly keeping this dynamic cycle of making changes automatically in the treatment as per the body's requirements, in a loop. This convergence of the human brain and the various computing technologies assisting the treatments, thus make it possible to achieve such intricate tasks with maximum efficiency. [4]

The concept of the Artificial Pancreas System can be considered as the best example of such an implementation in medicine. From an Industry 4.0 point of view, this system must be installed in the patient and as per the information and intervals fed to the system, it will release some pre-determined amount of insulin in the body at the required amount of time. This would be the entire basic working procedure of the system. But, as per Industry 5.0 ideologies, along with releasing insulin in the body it will also regulate the responses given by the body in return to the insulin amounts. All these responses are studied by this inter-disciplinary system and it can modify its pre-set programs if any changes are required in its working mechanism. After considering all the different factors governing the patients' life as a whole and the way in which the body would respond to them, the system then automatically finalizes an optimal treatment procedure which suits the best for that particular patient. The concept of a personal touch, that actually forms the basis of the medicine industry, is indeed a very crucial aspect and it can now be established through this intersection of AI and HI. [5]

\section{Industry 5.0: The art of building smart homes}

Not just limiting our scope to smart manufacturing and smart factories, we further put forth the idea of a smart home, a smart city and a smart society in general.

The concept of a dual-mesh network can be introduced which will enable contacting and communicating through wires or cables as well as through radiation by radio waves so that no two transmitting signals collide during the actual working. Instead of transmitting the message in the same traditional format across various networks, we can 
instead generate signals, spread it across all the nearby available devices where they would then receive these signals, interpret them and hence transmit the strongest message. [3]

\section{The future of smart homes}

Smart devices constitute a smart home, this concept is not only limited to setting alarms or generating reminders for specific tasks but expanding their reach and thinking beyond our scope is the need of the era. Taking the case of the security systems of our houses for instance, the systems installed in a smart home will not only make us aware of the intruders by ringing the alarm but also arrange for help by carrying out some responsive actions in such emergency situations. In the same way, the working of Refrigerators will also be beyond just regulating the temperature inside as per the surroundings whereas it would also let you know what type of food items are stored in it, which among them would perish easily on the basis for how long a food item has been kept inside and it would additionally also suggest ways of using those food items (recipes) effectively, at the earliest. In the same way, our disposal system of dustbins in smart homes can detect any improper segregation of wastes that is done and also suggest if there is any possibility of reusing or recycling some of the items thrown in the dustbin. [3]

A visionary once had said that 'this era requires thinking beyond transporting people by replacing it with teleportation'. With the introduction of Mixed Reality, self-contained holographic computers as its major highlight, would make teleportation possible. By bringing the best of both worlds (AR and VR) together featuring a wide Field of View, holograms could basically turn every imaginable thought into reality (into virtual reality). [10]

\section{Industry 5.0: Autonomous all the way}

In the past five years, autonomous drones have gone from "maybe possible" to "definitely possible" to "inevitable" to "how did anyone ever think this wasn't inevitable?" to the future of "now commercially available." Industry 4.0 introduced us with this drone concept which has almost become a daily chore for different activities, on the other hand Industry 5.0 offers us with the idea of self-driven autonomous drone system fed with a mapping application that makes them capable of taking the required response actions on their own in the given circumstances.

In this case considering aerial manoeuvres, let us take up a hypothetical situation of the surveillance of a fence by a drone. Instead of flying the drone all by ourselves, a program can be fed wherein the drone can take the decisions of the time and duration of the surveillance on its own. These drones can detect the presence of any suspicious person, observe their actions and in return send a message alert as a sign of the trespassing. Here, the job of Industry 5.0 and its offerings play a major role to change the old-school methods of drone flights, thus revolutionizing their capacity. [8]

Industry 5.0 has an overall impact not only on the industries but also on the lifestyles and working of humans as mentioned below:

The Economic Impact: Efforts yielding significant results in the achievement of a ZERO WASTE environment (Lean Management).

The Ecological Impact: The vigilant use of existing resources results in the further minimization of the exploitation of natural resources.

The Social Impact: A sense of mutual co-operation, understanding and growth between humans and machines are the driving forces in the implementation and execution of Industry 5.0. This results in transforming the working place into a great place to work in. [7] 


\section{Challenges}

In the near future, the adoption of Industry 5.0 would be more efficiently done if some of the estimated arising issues are resolved before hand. People working in an organization have to be assured of their safety and security while their mutual working with cobots. Along with workers, the stakeholders must also be taken into consideration as there might be some reluctance from their end to adopt changes in the overall working systems. Problems like over-production and a greater dependency on IT and electricity might cause a hindrance in the further industrial development. Also, the fact that this revolution in technology can be transformed into a bane for mankind instead of being a boon by some iniquitous people for their evil interests.

\section{A futuristic view of society 5.0}

With a view of not only having smart industries and smart homes, but we also visualize Industry 5.0 as a wholesome Smart City and Society. A smart society which experiences both the authenticity of nature as well as the advantages of technology, who with the help of software and machine learning can properly balance the ecosystem. An effort of leading the way back to humanity at work. A smart city is "a city that comprises six factors in its development policy: smart economy, smart mobility, smart environment, smart people, smart living, and smart governance". The collaboration of AI with HI will exponentially give rise to an efficient interwoven system of technologies with effective man-machine interaction, which becomes a major dynamic enabler. [9]

\section{Conclusions and Disclaimer}

Industry 5.0 offers us with the concepts of an efficient Supply-chain Management (SCM) system, Lean Management (LM), World class practices (WCP) along with the appropriate strategies needed for this to take place in a workplace. This collaborative working between humans and machines (automation) enables a customer centric, personalised, quality efficient and a productive industry culture.

The outcome of this revolution will not only be experienced by industries and businesses but also by the entire society and its people, resulting in the development of an entirely new version of lifestyle and working culture, bringing forth the best blend of man's cognitive ability and the ease of automation.[12]

\section{Declarations}

Ethics approval and consent to participate: Not applicable

Consent for publication: Not applicable

Availability of data and materials: All the publically accessible data are cited below.

Competing interests: The authors declare that they have no known competing financial interests or personal relationships that could have appeared to influence the work reported in this paper.

Funding: Not applicable

Author's contribution: Both the authors have equally done the work of the necessary information collection, the review article and the necessary format work. The study of all the topics mentioned here are done together by both author's. This review is done under the guidance of Dr. B. B. Ahuja (Director, COEP). 


\section{Acknowledgements}

All the opinions and information proposed in the following paper are solely of the writers' in accordance with the different sources of information studied and it has no motive of supporting any political view or does not have any governmental bias.

Guidance provided by our director Dr. B. B. Ahuja was greatly appreciated. Thanking him for his constant support and time.

\section{Citation}

- Industry 4.0 as a Part of Smart Cities (Michal Lom, Ondrej Pribyl, Miroslav Svitek, 2016)

- On the way from industry 4.0 to industry 5.0 (P.O. Skobelev, S.Yu. Borovik, 2017)

- The difference between autonomous and self-flying drones (Jonathan Feist, 2018)

- Industry 5.0 and sustainability, (Saeid Nahavandi, 2019).

\section{References}

[1] How Smart Homes Work - Molly Edmonds, Nathan Chandler https://home.howstuffworks.com/smart-home.htm

[2] Micron IT Director Tim Long https://www.micron.com/about https://ied.eu/project-updates/the-4industrial-revolutions/

[3] Industry 5.0 definition: Michael Rada https://medium.com/@michael.rada/industry-5-0-experts-rising$\underline{556253 \mathrm{a} 641 \mathrm{a} 3}$ 